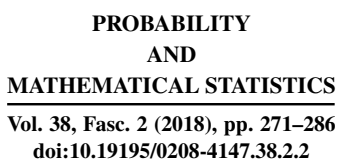

\title{
CONVERGENCE OF RANDOM OSCILLATORY INTEGRALS \\ IN THE PRESENCE OF LONG-RANGE DEPENDENCE AND APPLICATION TO HOMOGENIZATION
}

BY

ATEF LECHIHEB (TUNIS), IVAN NOURDIN (LUXEMBOURG), GUANGQU ZHENG (LAWRENCE), AND EZZEDINE HAOUALA (TUNIS)

\begin{abstract}
This paper deals with the asymptotic behavior of random oscillatory integrals in the presence of long-range dependence. As a byproduct, we solve the corrector problem in random homogenization of onedimensional elliptic equations with highly oscillatory random coefficients displaying long-range dependence, by proving convergence to stochastic integrals with respect to Hermite processes.
\end{abstract}

2010 AMS Mathematics Subject Classification: Primary: 60F05, 80M40; Secondary: 60H05, 60H20, 60G10, 60G18.

Key words and phrases: Elliptic equation, Hermite process, oscillatory integral, corrector, homogenization.

\section{MAIN RESULTS}

1.1. Convergence of random oscillatory integrals. One of our goals in the paper is to study, once properly normalized, the distributional convergence of some random oscillatory integrals of the form

$$
\int_{0}^{1} \Phi[g(x / \varepsilon)] h(x) d x,
$$

where

- $h \in C([0,1])$ is deterministic,

- $\{g(x)\}_{x \in \mathbb{R}_{+}}$is a certain centered stationary Gaussian process exhibiting long-range correlation,

- $\Phi \in L^{2}(\mathbb{R}, \nu)$ has Hermite rank $m \geqslant 1$ (with $\nu$ the standard Gaussian measure).

As we will see later, the main motivation of this study comes from the random corrector problem studied in [4]. 
Let us first introduce the Gaussian process $\{g(x)\}_{x \in \mathbb{R}_{+}}$we will deal with throughout all this paper. It is constructed as follows: $(1 / 2,1)$.

1. Let $m \in \mathbb{N}^{*}$ be fixed, let $H_{0} \in\left(1-\frac{1}{2 m}, 1\right)$, and set $H=1+m\left(H_{0}-1\right) \in$

2. Fix a slowly varying function $L:(0,+\infty) \rightarrow(0,+\infty)$ at $+\infty$, that is, consider a measurable and locally bounded function $L$ such that $L(\lambda x) / L(x) \rightarrow 1$ as $x \rightarrow+\infty$, for every $\lambda>0$. Assume furthermore that $L$ is bounded away from 0 and $+\infty$ on every compact subset of $(0,+\infty)$. (See [3] for more details on slowly varying functions.)

3. Let $e: \mathbb{R} \rightarrow \mathbb{R}$ be a square-integrable function such that

(3a) $\int_{\mathbb{R}} e(u)^{2} d u=1$;

(3b) $|e(u)| \leqslant C u^{H_{0}-3 / 2} L(u)$ for almost all $u>0$ and for some absolute constant $C$;

(3c) $e(u) \sim C_{0} u^{H_{0}-3 / 2} L(u)$, where $C_{0}=\left(\int_{0}^{\infty}\left(u+u^{2}\right)^{H_{0}-3 / 2} d u\right)^{-1 / 2}$;

(3d) there exists $0<\gamma<\min \left\{H_{0}-\left(1-\frac{1}{2 m}\right), 1-H_{0}\right\}$ such that

$$
\int_{-\infty}^{0}|e(u) e(x y+u)| d u=o\left(x^{2 H_{0}-2} L(x)^{2}\right) y^{2 H_{0}-2-2 \gamma}
$$

as $x \rightarrow \infty$, uniformly in $y \in(0, t]$ for each given $t>0$.

4. Finally, let $W$ be a two-sided Brownian motion.

Bearing all these ingredients in mind, we can now set, for $x \in \mathbb{R}_{+}$,

$$
g(x):=\int_{-\infty}^{\infty} e(x-\xi) d W_{\xi}
$$

REMARK 1.1. (i) Assumptions (3a) and 4 ensure that $\{g(x)\}_{x \in \mathbb{R}_{+}}$is a normalized centered Gaussian process.

(ii) Assumption (3b) controls $|e(u)|$ for small $u$, while assumption (3d) ensures that the "forward" contribution of $e(u)$ is ultimately negligible due to the following computation:

$$
\begin{aligned}
\mathbb{E}[g(s) g(s+x)] & =\int_{-\infty}^{\infty} e(s-\xi) e(s+x-\xi) d \xi=\int_{-\infty}^{\infty} e(u) e(u+x) d u \\
& =\int_{-\infty}^{0} e(u) e(u+x) d u+\int_{0}^{\infty} e(u) e(u+x) d u \\
& =o\left(x^{2 H_{0}-2} L(x)^{2}\right)+x \int_{0}^{\infty} e(x u) e(x u+x) d u
\end{aligned}
$$

(iii) Assumption (3c) ensures that the process $\{g(x)\}_{x \in \mathbb{R}_{+}}$exhibits the following asymptotic behavior:

$$
R_{g}(x):=\mathbb{E}[g(s) g(s+x)] \sim x^{2 H_{0}-2} L(x)^{2} \quad \text { as } x \rightarrow+\infty,
$$

see [12], equation (2.3). 
In Section B.], we will show that the random integral given by (ㅁ.J) exhibits the following asymptotic behavior as $\varepsilon \rightarrow 0$.

THEOREM 1.1. Let $g$ be the centered stationary Gaussian process defined by (․2.) and assume that $\Phi \in L^{2}(\mathbb{R}, \nu)$ has Hermite rank $m \geqslant 1$. Then, for any $h \in C([0,1])$, the following convergence in law holds:

$$
M_{h}^{\varepsilon}:=\frac{1}{\varepsilon d(1 / \varepsilon)} \int_{0}^{1} \Phi[g(x / \varepsilon)] h(x) d x \stackrel{\varepsilon \downarrow 0}{\longrightarrow} M_{h}^{0}:=\frac{V_{m}}{m !} \int_{0}^{1} h(x) d Z(x),
$$

where $Z$ is the mth order Hermite process defined by (2.4) below, and $d(\cdot)$ is defined by

$$
d(x)=\sqrt{\frac{m !}{H(2 H-1)}} x^{H} L(x)^{m} .
$$

As we already mentioned, the fine analysis of the asymptotic behavior of (IL.4) is motivated by the random corrector problem studied in [4]; it will be described below.

1.2. A motivating example. Theorem $\mathbb{L}$. appears to be especially useful and relevant in the study of the following homogenization problem. Consider the following one-dimensional elliptic equation displaying random coefficients:

$$
\left\{\begin{array}{l}
-\frac{d}{d x}\left(a(x / \varepsilon, \omega) \frac{d}{d x} u^{\varepsilon}(x, \omega)\right)=f(x), \quad x \in(0,1), \quad \varepsilon>0, \\
u^{\varepsilon}(0, \omega)=0, \quad u^{\varepsilon}(1, \omega)=b \in \mathbb{R} .
\end{array}\right.
$$

In (L.6), the random potential $\{a(x)\}_{x \in \mathbb{R}_{+}}$is assumed to be a uniformly bounded, positive $^{1}$ and stationary stochastic process, whereas the data $f$ is continuous. This model has received a lot of interests in the literature (see, e.g., [5], pp. 13-14).

Taking strong advantage of the fact that the ambient dimension is one, it is immediate to check that the solution to (1L.6) is given explicitly by

$$
u^{\varepsilon}(x, \omega)=c^{\varepsilon}(\omega) \int_{0}^{x} \frac{1}{a(y / \varepsilon, \omega)} d y-\int_{0}^{x} \frac{F(y)}{a(y / \varepsilon, \omega)} d y,
$$

where $F(x):=\int_{0}^{x} f(y) d y$ is the antiderivative of $f$ vanishing at zero, and where

$$
c^{\varepsilon}(\omega):=\left(b+\int_{0}^{1} \frac{F(y)}{a(y / \varepsilon, \omega)} d y\right)\left(\int_{0}^{1} \frac{1}{a(y / \varepsilon, \omega)} d y\right)^{-1} .
$$

\footnotetext{
${ }^{1}$ That is, there exists $r \in(0,1)$ such that $r \leqslant a(x) \leqslant r^{-1}$ for every $(x, \omega) \in \mathbb{R}_{+} \times \Omega$.
} 
Under suitable ergodic and stationary assumptions on $a$, the ergodic theorem applied to (ㅍ. 7 ) implies that $u^{\varepsilon}$ converges pointwise to $\bar{u}$ as $\varepsilon \rightarrow 0$, where

$$
\bar{u}(x)=\frac{c^{*} x}{a^{*}}-\int_{0}^{x} \frac{F(y)}{a^{*}} d y
$$

with $c^{*}:=b a^{*}+\int_{0}^{1} F(y) d y$ and

$$
a^{*}:=\frac{1}{\mathbb{E}[1 / a(0)]} .
$$

The above parameter $a^{*}$ is usually referred to as the effective diffusion coefficient in the literature, see e.g. [10]. It is also immediately checked that $\bar{u}$ is the unique solution to the following deterministic equation:

$$
\left\{\begin{array}{l}
-\frac{d}{d x}\left(a^{*} \frac{d}{d x} \bar{u}(x)\right)=f(x), \quad x \in(0,1), \\
\bar{u}(0)=0, \quad \bar{u}(1)=b .
\end{array}\right.
$$

Interested readers can refer to [2] for a recent review on models involving more general elliptic equations.

In this work, we address to the random corrector problem for (IL.6) in presence of long-range media, that is, we analyze the behavior of the random fluctuations between $u^{\varepsilon}$ and $\bar{u}$ when the random potential $a$ is obtained by means of a long-range process (see below for the details). Taking advantage of the explicit expressions for both (ㄴ.6) and (ㄷ.8), it is easy but crucial to observe that the random corrector $u^{\varepsilon}(x)-\bar{u}(x)$ can be fully expressed by means of random oscillatory integrals of the form

$$
\int_{0}^{1}\left[\frac{1}{a(y / \varepsilon)}-\frac{1}{a^{*}}\right] h(y) d y
$$

for some function $h$. Thus, the random corrector problem for (L.6) reduces in a careful analysis of the asymptotic behavior of random quantities of the form (L.9) as $\varepsilon \rightarrow 0$. To this aim, we need to give a precise description of the form of the process $a$.

Let $\nu$ denote the standard Gaussian measure on $\mathbb{R}$. Every $\Phi \in L^{2}(\mathbb{R}, \nu)$ admits the series expansion

$$
\Phi=\sum_{q=0}^{\infty} \frac{V_{q}}{q !} H_{q}, \quad \text { with } V_{q}:=\int_{\mathbb{R}} \Phi(x) H_{q}(x) \nu(d x),
$$

where $H_{q}(x)=(-1)^{q} \exp \left(x^{2} / 2\right) \frac{d^{q}}{d x^{q}} \exp \left(-x^{2} / 2\right)$ denotes the $q$ th Hermite polynomial. Recall that the integer $m_{\Phi}:=\inf \left\{q \geqslant 0: V_{q} \neq 0\right\}$ is called the Hermite 
rank of $\Phi$ (with the convention $\inf \emptyset=+\infty$ ). For any integer $m \geqslant 1$, we define $\mathscr{G}_{m}$ to be the collection of all square-integrable functions (with respect to the standard Gaussian measure on $\mathbb{R}$ ) that have Hermite rank $m$.

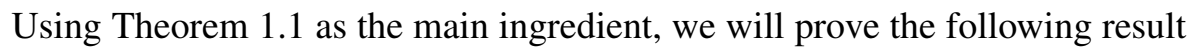
about the asymptotic behaviour of the random corrector associated with (L.6).

THEOREM 1.2. Fix an integer $m \geqslant 1$ as well as two real numbers $H_{0} \in$ $\left(1-\frac{1}{2 m}, 1\right)$ and $b \in \mathbb{R}$, and let $\{a(x)\}_{x \in \mathbb{R}_{+}}$be a uniformly bounded, positive and stationary stochastic process. Assume in addition that $q=\{q(x)\}_{x \in \mathbb{R}_{+}}$given by

$$
q(x)=\frac{1}{a(x)}-\frac{1}{a^{*}}, \quad \text { where } a^{*}:=1 / \mathbb{E}[1 / a(0)],
$$

has the form

$$
q(x)=\Phi(g(x))
$$

where $\Phi \in L^{2}(\mathbb{R}, \nu)$ belongs to $\mathscr{G}_{m}$ and $\{g(x)\}_{x \in \mathbb{R}_{+}}$is the Gaussian process given by (‥2). Finally, let $f:[0,1] \rightarrow \mathbb{R}$ be continuous and let us consider the solutions $u^{\varepsilon}$ and $\bar{u}$ of (ㄷ.6) and (․8) respectively. Then, for each $\varepsilon>0$, the random corrector $u^{\varepsilon}-\bar{u}$ is a continuous process on $[0,1]$. Moreover, we have the following convergence in law on $C([0,1])$ endowed with the supremum norm as $\varepsilon \rightarrow 0$ :

$$
\left\{\frac{u^{\varepsilon}(x)-\bar{u}(x)}{\varepsilon d(1 / \varepsilon)}\right\}_{x \in[0,1]} \Longrightarrow\left\{\frac{V_{m}}{m !} \int_{\mathbb{R}} F(x, y) d Z(y)\right\}_{x \in[0,1]},
$$

where dis given by ([1.5),

$$
\begin{gathered}
F(x)=\int_{0}^{x} f(y) d y, \quad c^{*}=a^{*} b+\int_{0}^{1} F(y) d y \\
F(x, y)=\left[c^{*}-F(y)\right] \mathbf{1}_{[0, x]}(y)+x\left(F(y)-\int_{0}^{1} F(z) d z-a^{*} b\right) \mathbf{1}_{[0,1]}(y),
\end{gathered}
$$

and $Z$ is the Hermite process of order $m$ and self-similar index

$$
H:=1+m\left(H_{0}-1\right) \in(1 / 2,1) .
$$

(The definition of $Z$ is given in Theorem 2.1 below.)

Note that it is not difficult to construct a process $a$ satisfying all the assumptions of Theorem 1.2. Indeed, bearing in mind the notation of Theorem L.2, we can write

$$
a(x)=\left(q(x)+\frac{1}{a^{*}}\right)^{-1}=\left(\Phi(g(x))+\frac{1}{a^{*}}\right)^{-1} .
$$


First, we note that since $g$ given by $(\mathbb{L} 2)$ is stationary, clearly the same holds for $a$, whatever the expression of $\Phi$. Second, given any fixed $a^{*}>0$, we can construct a bounded measurable function $\Phi \in \mathscr{G}_{2}$ with $\|\Phi\|_{\infty} \leqslant 1 /\left(2 a^{*}\right)$ as follows.

Let $h_{1}, h_{2}$ be two bounded measurable functions; then it is clear that they belong to $L^{2}(\mathbb{R}, \nu)$ and admit the series expansion

$$
h_{1}-\int_{\mathbb{R}} h_{1} d \nu=\sum_{k=1}^{\infty} a_{k} H_{k} \quad \text { and } \quad h_{2}-\int_{\mathbb{R}} h_{2} d \nu=\sum_{k=1}^{\infty} b_{k} H_{k},
$$

where the coefficients $a_{k}, b_{k}$ are defined in an obvious manner. Therefore, the function

$$
\Psi:=b_{1}\left(h_{1}-\int_{\mathbb{R}} h_{1} d \nu\right)-a_{1}\left(h_{2}-\int_{\mathbb{R}} h_{2} d \nu\right)
$$

is bounded and belongs to $\mathscr{G}_{2}$. Then we pick $\Phi=\Psi /\left(2 a^{*}\|\Psi\|_{\infty}\right) \in \mathscr{G}_{2}$. Therefore, $a(x)$ defined by ([L.53) satisfies

$$
0<\frac{2 a^{*}}{3} \leqslant a(x) \leqslant 2 a^{*} .
$$

Inductively, one can construct a bounded measurable $\Phi$ with Hermite rank $m \geqslant 3$ (by starting with two bounded functions in $\mathscr{G}_{m-1}$ ) such that the process $\{a(x), x \in$ $\mathbb{R}\}$ given in (11.13) satisfies (‥14).

Another possibility of constructing such a process $\{a(x), x \in \mathbb{R}\}$ is stated (more explicitly) as follows: let us fix $0<t_{1}<\ldots<t_{m}$ and consider the unique $(m+1)$-tuple $\left(b_{0}, \ldots, b_{m}\right)$ satisfying

$$
\left\{\begin{array}{l}
\sum_{l=0}^{m} b_{l} e^{-k t_{l}}=0 \quad \text { for all } k \in\{0, \ldots, m-1\} \\
\sum_{l=0}^{m} b_{l} e^{-m t_{l}}=1 .
\end{array}\right.
$$

(The existence and uniqueness of a solution to (‥5) is a consequence of a Vandermonde determinant.) Now, consider any measurable function $\psi$ satisfying

$$
0 \leqslant \psi \leqslant \frac{1}{2 a^{*} \sum_{l=0}^{m}\left|b_{l}\right|} .
$$

Since $\psi$ belongs obviously to $L^{2}(\mathbb{R}, \nu)$, it may be expanded in Hermite polynomials as $\psi=\sum_{k=0}^{\infty} a_{k} H_{k}$. We assume moreover that $a_{m} \neq 0$. (The existence of $\psi$ satisfying both (‥16) and $a_{m} \neq 0$ is clear by a contradiction argument.) Now, let

$$
\Phi=\sum_{l=0}^{m} b_{l} P_{t_{l}} \psi
$$


where $P_{t} \psi(x)=\int_{\mathbb{R}} \psi\left(e^{-t} x+\sqrt{1-e^{-2 t}} y\right) \nu(d y)$ is the classical Ornstein-Uhlenbeck semigroup. Due to (‥5), it is readily checked that the expansion of $\Phi$ is

$$
\Phi=a_{m} H_{m}+\sum_{k=m+1}^{\infty}\left\{\sum_{l=0}^{m} b_{l} e^{-k t_{l}}\right\} a_{k} H_{k}
$$

so that $\Phi \in \mathscr{G}_{m}$. Moreover,

$$
\|\Phi\|_{\infty} \leqslant \sum_{l=0}^{m}\left|b_{l}\right|\left\|P_{t_{l}} \psi\right\|_{\infty} \leqslant\|\psi\|_{\infty} \sum_{l=0}^{m}\left|b_{l}\right| \leqslant \frac{1}{2 a^{*}}
$$

and $a$ given by (․․3) is positive and bounded. So, the existence of a process $a$ satisfying all the assumptions of Theorem $\mathbb{L}$.2 is shown.

Theorem L.2 should be seen as an extension of and a unified approach to the main results of [4], and it contains these results as particular cases. More precisely, the case where the Hermite rank of $\Phi$ is $m=1$ corresponds to Theorem 2.5 in [1]] and involves the fractional Brownian motion in the limit, whereas the case where the Hermite rank of $\Phi$ is $m=2$ corresponds to Theorem 2.2 in [4] and involves the Rosenblatt process in the limit. Also, in their last section (entitled Conclusions and further discussion), the authors of [4] pointed out that "it is natural to ask what would happen if the Hermite rank of $\Phi$ was greater than 2". Our Theorem 1.2 answers this question by showing (as guessed by the authors of [4]) that, in the case $m \geqslant 3$, the limit takes the form of an integral with respect to the Hermite process of order $m$. Finally, we would like to emphasize that our Theorem 1.2 , even in the cases $m=1$ and $m=2$, is a strict extension of the results of [4], as we allow the possibility to deal with a slowly varying function $L$. That being said, our proof of Theorem [L.2 is exclusively based on the ideas and results contained in the seminal paper [12] and follows the strategy developed in [4]. In higher dimension, it is usually very hard to study the corrector theory due to the lack of the explicit form of the solution. In the recent papers [8], [9], the authors considered the discretized version of the corrector problem in higher dimension and were able to study the scaling limit to some Gaussian fields. For more details, we refer the interested readers to these two papers and the references therein.

The rest of the paper is organized as follows. In Section 2, we give some preliminary results divided into several subsections. Section 3 contains the proof of Theorems [.2 and ‥1.

\section{PRELIMINARY RESULTS}

Throughout this section, we let all the notation and assumptions of Sections $\square$ and ए.2 prevail. 
2.1. Asymptotic behavior of the covariance function of $q$. For $x \in \mathbb{R}$, set $R_{q}(x)=\mathbb{E}[q(0) q(x)]$. Also, recall that $m$ is the Hermite rank of $\Phi$. Then, proceeding in similar lines to those in Lemma 2.1 of [4], one can show that

$$
\left|R_{q}(x)\right|=\left(o(1)+V_{m}^{2} / m !\right) L(|x|)^{2 m}|x|^{-2(1-H)}
$$

as $|x| \rightarrow+\infty$. Here $o(1)$ means that the term converges to zero when $x \rightarrow \infty$.

The asymptotic relation (2.1) implies the existence of some absolute constant $C$ satisfying

$$
\left|R_{q}(x)\right| \leqslant C L(|x|)^{2 m}|x|^{-2(1-H)}
$$

for any $x \neq 0$.

2.2. Taqqu's theorem and convergence to the Hermite process $Z$. Recall $d(x)$ from ([L.5). Its main property is that the variance of $\frac{1}{d(x)} \int_{0}^{x} H_{m}(g(y)) d y$ is asymptotically equal to one as $x \rightarrow+\infty$.

The following result, due to Taqqu in 1979, is the key ingredient in our proofs.

THEOREM 2.1 ([12], Lemma 5.3). Let us assume $\Phi \in \mathscr{G}_{m}$ and let $g$ be given by (․2). Then, as $T \rightarrow+\infty$, the process

$$
Y_{T}(x)=\frac{1}{d(T)} \int_{0}^{T x} \Phi[g(y)] d y, \quad x \in \mathbb{R}_{+}
$$

converges to $\left(V_{m} / m !\right) Z(x)$ in the sense of finite-dimensional distributions, where the mth order Hermite process $Z$ with self-similar index $H=m\left(H_{0}-1\right)+1$ is defined by

(2.4) $Z(x)$

$$
=K\left(m, H_{0}\right)\left\{\int_{-\infty}^{\infty} d B_{\xi_{1}} \int_{-\infty}^{\xi_{1}} d B_{\xi_{2}} \ldots \int_{-\infty}^{\xi_{m-1}} d B_{\xi_{m}} \int_{0}^{x} \prod_{i=1}^{m}\left(s-\xi_{i}\right)^{H_{0}-3 / 2} \mathbf{1}_{\left(\xi_{i}<s\right)} d s\right\},
$$

where

$$
K\left(m, H_{0}\right):=\sqrt{\frac{m ! H(2 H-1)}{\left(\int_{0}^{\infty}\left(u+u^{2}\right)^{H_{0}-3 / 2} d u\right)^{m}}}
$$

is the normalizing constant such that $\mathbb{E}\left[Z(1)^{2}\right]=1$. (See [12], equation (1.6).)

Note that $Z(x)$ lives in the Wiener chaos of order $m$, which is non-Gaussian unless $m=1$ or $x=0$. 
2.3. Wiener integral with respect to $Z$. Let $Z$ be given as above and let $\mathcal{E}$ be the set of elementary (deterministic) functions, that is, the set of functions $h$ of the form

$$
h(x)=\sum_{k=1}^{\ell} a_{k} \mathbf{1}_{\left(t_{k}, t_{k+1}\right]}(x)
$$

with $\ell \in \mathbb{N}^{*}, a_{k} \in \mathbb{R}, t_{k}<t_{k+1}$. For such $h$, we define the Wiener integral with respect to $Z$ in the usual way, as a linear functional over $\mathcal{E}$ :

$$
\int_{\mathbb{R}} h(x) d Z(x)=\sum_{k=1}^{\ell} a_{k}\left[Z\left(t_{k+1}\right)-Z\left(t_{k}\right)\right] .
$$

One can easily verify that this definition is independent of choices of representation for elementary functions. Now we introduce the space of (deterministic) integrands for this Wiener integral:

$$
\Lambda^{H}=\left\{f: \mathbb{R} \longrightarrow \mathbb{R}\left|\int_{\mathbb{R}} \int_{\mathbb{R}} f(u) f(v)\right| u-\left.v\right|^{2 H-2} d u d v<+\infty\right\},
$$

equipped with the norm

$$
\|f\|_{\Lambda^{H}}^{2}=H(2 H-1) \int_{\mathbb{R}} \int_{\mathbb{R}} f(u) f(v)|u-v|^{2 H-2} d u d v .
$$

When $h \in \mathcal{E}$, it is straightforward to check the following isometry property:

$$
\mathbb{E}\left[\left(\int_{\mathbb{R}} h(x) d Z(x)\right)^{2}\right]=\|h\|_{\Lambda^{H}}^{2}
$$

As a consequence, one can define the Wiener integral $\int_{\mathbb{R}} f(x) d Z(x)$ for any $f \in$ $\Lambda^{H}$ by a usual approximation procedure.

It is well known by now (thanks to [I]] ) that $\left(\Lambda^{H},\|\cdot\|_{\Lambda^{H}}\right)$ is a Hilbert space that contains distributions in the sense of Schwartz. To overcome this problem, we shall restrict ourselves to the proper subspace

$$
\left|\Lambda^{H}\right|=\left\{f: \mathbb{R} \rightarrow \mathbb{R}\left|\int_{\mathbb{R}} \int_{\mathbb{R}}\right| f(u) f(v)|| u-\left.v\right|^{2 H-2} d u d v<+\infty\right\}
$$

equipped with the norm

$$
\|f\|_{\left|\Lambda^{H}\right|}^{2}=H(2 H-1) \int_{\mathbb{R}} \int_{\mathbb{R}}|f(u) f(v) \| u-v|^{2 H-2} d u d v .
$$

We then have (see [11], Proposition 4.2)

$$
L^{1}(\mathbb{R}) \cap L^{2}(\mathbb{R}) \subset L^{1 / H}(\mathbb{R}) \subset\left|\Lambda^{H}\right| \subset \Lambda^{H} .
$$


Moreover, $\left(\left|\Lambda^{H}\right|,\|\cdot\|_{\left|\Lambda^{H}\right|}\right)$ is a Banach space in which the set $\mathcal{E}$ is dense. So for $h \in\left|\Lambda^{H}\right|$, we can define

$$
\int_{\mathbb{R}} h(x) d Z(x)=\lim _{n \rightarrow+\infty} \int_{\mathbb{R}} h_{n}(x) d Z(x),
$$

where $\left(h_{n}\right)$ is any sequence of $\mathcal{E}$ converging to $h$ in $\left(\left|\Lambda^{H}\right|,\|\cdot\|_{\left|\Lambda^{H}\right|}\right)$; the convergence in (2.8) holds in $L^{2}(\Omega)$.

For a detailed account of this integration theory, one can refer to [U], [II]].

2.4. Some facts about slowly varying functions. Let $L:(0,+\infty) \rightarrow(0,+\infty)$ be a slowly varying function at $+\infty$ and $\alpha>0$. It is well known (see [3], Proposition 1.3.6(v)) that

$$
x^{\alpha} L(x) \rightarrow+\infty \quad \text { and } \quad x^{-\alpha} L(x) \rightarrow 0
$$

as $x \rightarrow+\infty$. In particular, one can deduce that

$$
\lim _{\varepsilon \downarrow 0} \varepsilon^{1-H} L(1 / \varepsilon)^{m}=0 .
$$

The following result is known as Potter's theorem (see [3], Theorem 1.5.6(ii)).

THEOREM 2.2. Let $L:(0,+\infty) \rightarrow(0,+\infty)$ be a slowly varying function at $+\infty$ such that it is bounded away from 0 and $+\infty$ on every compact subset of $(0,+\infty)$. Then for any $\delta>0$ there exists some constant $C=C(\delta)$ such that

$$
\frac{L(y)}{L(x)} \leqslant C \max \left\{(x / y)^{\delta},(y / x)^{\delta}\right\} \quad \text { for any } x, y \in(0,+\infty) .
$$

\section{PROOFS OF THE MAIN RESULTS}

3.1. Proof of Theorem 1.1. First recall that a typical function $h$ in $\mathcal{E}$ has the form

$$
h(x)=\sum_{\ell=1}^{n} a_{\ell} \mathbf{1}_{\left(t_{\ell}, t_{\ell+1}\right]}(x), \quad t_{\ell}<t_{\ell+1}, \quad a_{\ell} \in \mathbb{R}, \quad \ell=1, \ldots, n .
$$

For such a simple function $h$, we deduce from Taqqu's theorem (Theorem 2.1]) that

$$
\begin{aligned}
M_{h}^{\varepsilon} & =\frac{1}{\varepsilon d(1 / \varepsilon)} \int_{\mathbb{R}} q(x / \varepsilon) \sum_{\ell=1}^{n} a_{\ell} \mathbf{1}_{\left(t_{\ell}, t_{\ell+1}\right]}(x) d x \\
& =\sum_{\ell=1}^{n} a_{\ell} \frac{1}{d(1 / \varepsilon)}\left(\int_{0}^{t_{\ell+1} / \varepsilon} \Phi(g(x)) d x-\int_{0}^{t_{\ell} / \varepsilon} \Phi(g(x)) d x\right) \\
& \stackrel{\varepsilon \rightarrow 0}{\longrightarrow} \frac{V_{m}}{m !} \sum_{\ell=1}^{n} a_{\ell}\left[Z\left(t_{\ell+1}\right)-Z\left(t_{\ell}\right)\right]=\frac{V_{m}}{m !} \int_{\mathbb{R}} h(x) d Z(x) .
\end{aligned}
$$

This proves (ㄴ.4) for simple functions $h \in \mathcal{E}$. 
Let us now consider $h \in C([0,1])$. It is easy to see that there exists a sequence $\left(h_{n}\right) \subset \mathcal{E}$ such that

$$
\lim _{n \rightarrow+\infty}\left\|h_{n}-h\right\|_{\infty}=0
$$

Let us fix a number $\zeta \in(0,1)$ and show the convergence in $L^{2}(\Omega)$ of $M_{h_{n}}^{\varepsilon}$, uniformly in $\varepsilon \in(0, \zeta)$. First, one can write

$$
\begin{aligned}
\sup _{\varepsilon \in(0, \zeta)} \mathbb{E}\left[\left|M_{h_{n}}^{\varepsilon}-M_{h}^{\varepsilon}\right|^{2}\right] \\
\quad=\sup _{\varepsilon \in(0, \zeta)} \frac{1}{\varepsilon^{2} d(1 / \varepsilon)^{2}} \mathbb{E}\left[\left|\int_{0}^{1} q(x / \varepsilon)\left[h_{n}(x)-h(x)\right] d x\right|^{2}\right] \\
\quad \leqslant\left\|h_{n}-h\right\|_{\infty}^{2} \sup _{\varepsilon \in(0, \zeta)} \frac{1}{\varepsilon^{2} d(1 / \varepsilon)^{2}} \int_{\mathbb{R}^{2} \backslash D}\left|R_{q}\left(\frac{y-x}{\varepsilon}\right)\right| d x d y
\end{aligned}
$$

where $D=\left\{(x, y) \in[0,1]^{2}: x=y\right\}$ is a negligible subset of $\mathbb{R}^{2}$. By (2.2),

$$
\left|R_{q}\left(\frac{y-x}{\varepsilon}\right)\right| \leqslant \operatorname{Cst} L\left(\left|\frac{y-x}{\varepsilon}\right|\right)^{2 m}\left|\frac{y-x}{\varepsilon}\right|^{-2(1-H)} \quad \text { for all }(x, y) \in \mathbb{R}^{2} \backslash D \text {. }
$$

Second, with $\beta>0$ small enough such that $2 m \beta+2(1-H) \in(0,1)$, we have

$$
\begin{aligned}
& \sup _{\varepsilon \in(0, \zeta)} \frac{1}{X(\varepsilon)^{2}} \int_{[0,1]^{2} \backslash D}\left|R_{q}\left(\frac{y-x}{\varepsilon}\right)\right| d x d y \\
& \quad \leqslant \text { Cst } \sup _{\varepsilon \in(0, \zeta)} \int_{[0,1]^{2} \backslash D}\left\{\frac{L(|(x-y) / \varepsilon|)}{L(1 / \varepsilon)}\right\}^{2 m}|x-y|^{-2(1-H)} d x d y \\
& \quad \leqslant \text { Cst } \int_{[0,1]^{2} \backslash D}|x-y|^{-2 m \beta-2(1-H)} d x d y \\
& \quad<+\infty
\end{aligned}
$$

where the second inequality follows from Theorem 2.2. It is now clear that, indeed,

$$
\lim _{n \rightarrow+\infty} \sup _{\varepsilon \in(0, \zeta)} \mathbb{E}\left[\left|M_{h_{n}}^{\varepsilon}-M_{h}^{\varepsilon}\right|^{2}\right]=0 .
$$

To conclude, let $d(\cdot, \cdot)$ denote any distance metrizing the convergence in distribution between real-valued random variables (for instance, the Fortet-Mourier distance). For $h \in C([0,1])$ and $\left(h_{n}\right) \subset \mathcal{E}$ converging to $h$, one can write, for any $\varepsilon>0$ and $n \in \mathbb{N}$ :

$$
d\left(M_{h}^{\varepsilon}, M_{h}^{0}\right) \leqslant d\left(M_{h}^{\varepsilon}, M_{h_{n}}^{\varepsilon}\right)+d\left(M_{h_{n}}^{\varepsilon}, M_{h_{n}}^{0}\right)+d\left(M_{h_{n}}^{0}, M_{h}^{0}\right) .
$$


Fix $\eta>0$. By (B.2), one can choose $n$ big enough so that, for any $\varepsilon \in(0, \zeta)$, both $d\left(M_{h}^{\varepsilon}, M_{h_{n}}^{\varepsilon}\right)$ and $d\left(M_{h_{n}}^{0}, M_{h}^{0}\right)$ are less than $\eta / 3$. It remains to choose $\varepsilon>0$ small enough so that $d\left(M_{h_{n}}^{\varepsilon}, M_{h_{n}}^{0}\right)$ is less than $\eta / 3$ (by (L.4) for the simple function $\left.h_{n} \in \mathcal{E}\right)$, to conclude that (‥4) holds true for any continuous function $h$.

REMARK 3.1. Clearly, the above result still holds true for any function $h$ that is continuous except at finitely many points. Note also that the function $\Phi \in \mathscr{G}_{m}$ is not necessarily bounded in Theorem $\mathbb{L}$.1].

3.2. Proof of Theorem 1.2. The proof is divided into five steps. We write

$$
X(\varepsilon)=\varepsilon d(1 / \varepsilon)=\sqrt{\frac{m !}{H(2 H-1)}} \varepsilon^{1-H} L(1 / \varepsilon)^{m} .
$$

(a) Preparation. Following [4], especially identities (5.1) and (5.19) therein, we first rewrite the rescaled corrector as follows:

$$
\frac{u^{\varepsilon}(x)-\bar{u}(x)}{X(\varepsilon)}=\mathcal{U}^{\varepsilon}(x)+\underbrace{\frac{1}{X(\varepsilon)} r^{\varepsilon}(x)+\frac{1}{X(\varepsilon)} \rho^{\varepsilon} \frac{x}{a^{*}}}_{=: \mathcal{R}^{\varepsilon}(x)},
$$

where

$$
\begin{gathered}
\mathcal{U}^{\varepsilon}(x)=\frac{1}{X(\varepsilon)} \int_{\mathbb{R}} F(x, y) q(y / \varepsilon) d y, \\
r^{\varepsilon}(x)=\left(c^{\varepsilon}-c^{*}\right) \int_{0}^{x} q(y / \varepsilon) d y,
\end{gathered}
$$

and

$$
\begin{aligned}
\rho^{\varepsilon}:= & \frac{a^{*}}{\int_{0}^{1} a(y / \varepsilon)^{-1} d y}\left[\left(a^{*} b+\int_{0}^{1} F(y) d y\right)\right. \\
& \left(\int_{0}^{1} q(y / \varepsilon) d y\right)^{2} \\
& \left.-\int_{0}^{1} F(y) q(y / \varepsilon) d y \int_{0}^{1} q(y / \varepsilon) d y\right] .
\end{aligned}
$$

Now, let us first show the weak convergence of $\mathcal{U}^{\varepsilon}$ to $\mathcal{U}$ in $C([0,1])$ and then prove that $\mathcal{R}^{\varepsilon}$ is a remainder. To prove the first claim, we start by establishing the f.d.d. convergence and then prove the tightness.

(b) Convergence of finite-dimensional distributions of $\mathcal{U}^{\varepsilon}$. For $x_{1}, \ldots, x_{n} \in \mathbb{R}$ and $\lambda_{1}, \ldots, \lambda_{n} \in \mathbb{R}(n \geqslant 1)$, we have

$$
\sum_{k=1}^{n} \lambda_{k} \mathcal{U}^{\varepsilon}\left(x_{k}\right)=\frac{1}{X(\varepsilon)} \int_{\mathbb{R}} \sum_{k=1}^{n} \lambda_{k} F\left(x_{k}, y\right) q(y / \varepsilon) d y .
$$


Note that the function $\sum_{k=1}^{n} \lambda_{k} F\left(x_{k}, \cdot\right)$ has at most finitely many discontinuities. Thus, Theorem $\mathbb{\square}$ ] and Remark B.] imply that $\sum_{k=1}^{n} \lambda_{k} \mathcal{U}^{\varepsilon}\left(x_{k}\right)$ converges in distribution to $\sum_{k=1}^{n} \lambda_{k} \mathcal{U}\left(x_{k}\right)$, yielding the desired convergence of finite-dimensional distributions.

(c) Tightness of $\mathcal{U}^{\varepsilon}$. We check Kolmogorov's criterion ([6], Corollary 16.9). First observe that $\mathcal{U}^{\varepsilon}(0)=0$. Now, fix $0 \leqslant u<v \leqslant 1$, and set $F_{1}(y)=c^{*}-$ $F(y)$ and $F_{2}(y)=F(y)-\int_{0}^{1} F(t) d t-a^{*} b$, so that $F(x, y)=F_{1}(y) \mathbf{1}_{[0, x]}(y)+$ $x F_{2}(y) \mathbf{1}_{[0,1]}(y)$. Then

$$
\begin{aligned}
& \text { (3.4) } \begin{aligned}
& \mathbb{E}\left(\left|\mathcal{U}^{\varepsilon}(u)-\mathcal{U}^{\varepsilon}(v)\right|^{2}\right) \\
&= \mathbb{E}\left[\frac{1}{X(\varepsilon)^{2}}\left|\int_{0}^{1} \mathbf{1}_{(u, v]}(y) q(y / \varepsilon) F_{1}(y) d y+(v-u) \int_{0}^{1} q(y / \varepsilon) F_{2}(y) d y\right|^{2}\right] \\
& \leqslant \frac{2}{X(\varepsilon)^{2}} \mathbb{E}\left[\left|\int_{0}^{1} \mathbf{1}_{(u, v]}(y) q(y / \varepsilon) F_{1}(y) d y\right|^{2}+\left|(v-u) \int_{0}^{1} q(y / \varepsilon) F_{2}(y) d y\right|^{2}\right] \\
& \leqslant \frac{2}{X(\varepsilon)^{2}} \int_{u}^{v} \int_{u}^{v} F_{1}(x) F_{1}(y) R_{q}\left(\frac{y-x}{\varepsilon}\right) d x d y \\
&+\frac{2(v-u)^{2}}{X(\varepsilon)^{2}} \int_{0}^{1} \int_{0}^{1} F_{2}(x) F_{2}(y) R_{q}\left(\frac{y-x}{\varepsilon}\right) d x d y .
\end{aligned}
\end{aligned}
$$

Note that $F_{2}$ is bounded on $[0,1]$. Therefore, as far as the second term in the last inequality in (B.4) is concerned, one can write, using Potter's theorem as in the proof of Theorem LI,

$$
\sup _{\varepsilon \in(0, \zeta)}\left|\frac{(v-u)^{2}}{X(\varepsilon)^{2}} \int_{0}^{1} \int_{0}^{1} F_{2}(x) F_{2}(y) R_{q}\left(\frac{y-x}{\varepsilon}\right) d x d y\right| \leqslant \operatorname{Cst}(v-u)^{2} .
$$

Now, let us consider the first term in the last inequality in (B.4). Similarly,

$$
\begin{aligned}
& \sup _{\varepsilon \in(0, \zeta)} \frac{1}{X(\varepsilon)^{2}}\left|\int_{u}^{v} \int_{u}^{v} F_{1}(x) F_{1}(y) R_{q}\left(\frac{y-x}{\varepsilon}\right) d x d y\right| \\
& \leqslant \mathrm{Cst} \sup _{\varepsilon \in(0, \zeta)} \frac{1}{X(\varepsilon)^{2}} \int_{u}^{v} \int_{u}^{v}\left|R_{q}\left(\frac{y-x}{\varepsilon}\right)\right| d x d y \quad \text { (since } F_{1} \text { is bounded) } \\
& \leqslant \mathrm{Cst} \sup _{\varepsilon \in(0, \zeta)} \frac{1}{L(1 / \varepsilon)^{2 m}} \int_{u}^{v} \int_{u}^{v} L(|y-x| / \varepsilon)^{2 m} \frac{d x d y}{|y-x|^{2(1-H)}} \\
& \left.\leqslant \mathrm{Cst} \int_{u}^{v} \int_{u}^{v}|y-x|^{-2(1-H)-2 m \beta} d y d x \quad \text { (as in (B. (1) ) }\right) \\
& =\mathrm{Cst}(v-u)^{2-2 m \beta-2(1-H)} .
\end{aligned}
$$

Since $2-2 m\left(1-H_{0}\right)-2 m \beta>1$, this proves the tightness of $\left(\mathcal{U}^{\varepsilon}\right)_{\varepsilon}$ by means of the usual Kolmogorov's criterion. 
(d) Control on the remainder term $\mathcal{R}^{\varepsilon}$ in (B.3). We shall prove that the process $\mathcal{R}^{\varepsilon}$ converges in probability to zero in $C([0,1])$. First we claim that if $G \in$ $C([0,1])$, then there exists some constant $C=C(G)$ such that

$$
\sup _{x \in[0,1]} \mathbb{E}\left[\left(\int_{0}^{x} q(y / \varepsilon) G(y) d y\right)^{2}\right] \leqslant C X(\varepsilon)^{2} .
$$

Indeed, the same argument we used for obtaining (B.5) works here as well, so we get

$$
\begin{aligned}
\sup _{x \in[0,1]} \mathbb{E}\left[\left(\int_{0}^{x} q(y / \varepsilon) G(y) d y\right)^{2}\right] \\
\quad \leqslant\|G\|_{\infty}^{2} \int_{[0,1]^{2}}\left|R_{q}(|y-z| / \varepsilon)\right| d y d z \\
\quad \leqslant\|G\|_{\infty}^{2} X(\varepsilon)^{2}\left(\sup _{\varepsilon \in(0, \zeta)} \frac{1}{X(\varepsilon)^{2}} \int_{[0,1]^{2}}\left|R_{q}(|y-z| / \varepsilon)\right| d y d z\right) \\
\quad \leqslant \operatorname{Cst} X(\varepsilon)^{2},
\end{aligned}
$$

where the last inequality follows from (B.]).

Now, let us consider $\mathcal{R}^{\varepsilon}$ :

(i) Due to the explicit expression of $\rho^{\varepsilon}$, it follows from (B.7), the fact that $a$ is bounded from below and the Cauchy-Schwarz inequalities that

$$
\begin{aligned}
& \mathbb{E}\left[\left|\rho^{\varepsilon}\right|\right] \\
\leqslant & \operatorname{Cst}\left\{\left\|\int_{0}^{1} q(y / \varepsilon) d y\right\|_{L^{2}(\Omega)}^{2}+\left\|\int_{0}^{1} F(y) q(y / \varepsilon) d y\right\|_{L^{2}(\Omega)}\left\|\int_{0}^{1} q(y / \varepsilon) d y\right\|_{L^{2}(\Omega)}\right\} \\
\leqslant & \operatorname{Cst} X(\varepsilon)^{2} .
\end{aligned}
$$

(ii) Observe that

$$
\begin{aligned}
c^{\varepsilon}-c^{*} & =a^{*} \int_{0}^{1}\left(F(y)-\int_{0}^{1} F(t) d t-b a^{*}\right) q(y / \varepsilon) d y+\rho^{\varepsilon} \\
& =: \int_{0}^{1} \widehat{F}(y) q(y / \varepsilon) d y+\rho^{\varepsilon} .
\end{aligned}
$$

Then

$$
\begin{aligned}
& \sup _{x \in[0,1]} \mathbb{E}\left[\left|r^{\varepsilon}(x)\right|\right]=\sup _{x \in[0,1]} \mathbb{E}\left[\left|\left(c^{\varepsilon}-c^{*}\right) \int_{0}^{x} q(y / \varepsilon) d y\right|\right] \\
& \leqslant \sup _{x \in[0,1]} \mathbb{E}\left[\left|\int_{0}^{1} \widehat{F}(y) q(y / \varepsilon) d y \int_{0}^{x} q(y / \varepsilon) d y\right|\right]+\operatorname{Cst} \mathbb{E}\left[\left|\rho^{\varepsilon}\right|\right] \leqslant \operatorname{Cst} X(\varepsilon)^{2} .
\end{aligned}
$$


Therefore, as $\varepsilon \rightarrow 0$ we have, by (2.9),

$$
\sup _{x \in[0,1]} \mathbb{E}\left[\left|\mathcal{R}^{\varepsilon}(x)\right|\right] \leqslant \operatorname{Cst} X(\varepsilon) \rightarrow 0 .
$$

In particular, $\left\{\mathcal{R}^{\varepsilon}(x), x \in[0,1]\right\}$ converges to zero in the sense of finite-dimensional distributions. Now, let us check the tightness of $\left(\mathcal{R}^{\varepsilon}\right)_{\varepsilon}$. Note that $\mathcal{R}^{\varepsilon}(0)=0$ and that, for $0 \leqslant u<v \leqslant 1$,

$$
\begin{aligned}
\left\|\mathcal{R}^{\varepsilon}(u)-\mathcal{R}^{\varepsilon}(v)\right\|_{L^{2}(\Omega)}^{2} & \\
\leqslant & \frac{2}{X(\varepsilon)^{2}}\left\{\left\|r^{\varepsilon}(u)-r^{\varepsilon}(v)\right\|_{L^{2}(\Omega)}^{2}+\frac{2(u-v)^{2}}{\left|a^{*}\right|^{2}} \mathbb{E}\left[\left|\rho^{\varepsilon}\right|^{2}\right]\right\} \\
\leqslant & \frac{2}{X(\varepsilon)^{2}}\left\|r^{\varepsilon}(u)-r^{\varepsilon}(v)\right\|_{L^{2}(\Omega)}^{2} \\
& +\operatorname{Cst} \frac{(u-v)^{2}}{X(\varepsilon)^{2}} \mathbb{E}\left[\left|\rho^{\varepsilon}\right|\right] \quad\left(\text { since } \rho^{\varepsilon}\right. \text { is uniformly bounded) } \\
\leqslant & \frac{2}{X(\varepsilon)^{2}}\left\|r^{\varepsilon}(u)-r^{\varepsilon}(v)\right\|_{L^{2}(\Omega)}^{2}+\operatorname{Cst}(u-v)^{2} \quad \text { (by point (i) above) } \\
\leqslant & \operatorname{Cst} \frac{1}{X(\varepsilon)^{2}} \int_{[u, v]^{2}}|R((y-z) / \varepsilon)| d y d z \\
& +\operatorname{Cst}(u-v)^{2} \quad\left(\text { since } c^{\varepsilon}-c^{*}\right. \text { is uniformly bounded) } \\
\leqslant & \operatorname{Cst}(v-u)^{2-2(1-H)-2 m \beta}+\operatorname{Cst}(v-u)^{2},
\end{aligned}
$$

where the last inequality follows from the same arguments as in (B.6). Therefore, $\mathcal{R}^{\varepsilon}$ converges in distribution to zero, as $\varepsilon \downarrow 0$, so it converges in probability to zero.

(e) Conclusion. Combining the results of (a) to (d), we conclude the proof of Theorem 12 by evoking the Slutsky lemma.

\section{REFERENCES}

[1] G. Bal, J. Garnier, S. Motsch, and V. Perrier, Random integrals and correctors in homogenization, Asymptot. Anal. 59 (1-2) (2008), pp. 1-26.

[2] G. Bal and Y. Gu, Limiting models for equations with large random potential: A review, Commun. Math. Sci. 13 (3) (2015), pp. 729-748.

[3] N. H. Bingham, C. M. Goldie, and J. L. Teugels, Regular Variation, Cambridge University Press, Cambridge 1989.

[4] Y. Gu and G. B al, Random homogenization and convergence to integrals with respect to the Rosenblatt process, J. Differential Equations 253 (4) (2012), pp. 1069-1087.

[5] V. V. Jikov, S. M. Kozlov, and O. A. Olě̆nik, Homogenization of Differential Operators and Integral Functionals, Springer, Berlin 1994.

[6] O. Kallenberg, Foundations of Modern Probability, second edition, Springer, New York 2002.

[7] M. Maejima and C. A. Tudor, Wiener integrals with respect to the Hermite process and a non-central limit theorem, Stoch. Anal. Appl. 25 (5) (2007), pp. 1043-1056. 
[8] J. Mourrat and J. Nolen, Scaling limit of the corrector in stochastic homogenization, Ann. Appl. Probab. 27 (2) (2017), pp. 944-959.

[9] J. Mourrat and F. Otto, Correlation structure of the corrector in stochastic homogenization, Ann. Probab. 44 (5) (2016), pp. 3207-3233.

[10] G. C. Papanicolaou and S. R. S. Varadhan, Boundary value problems with rapidly oscillating random coefficients, in: Proceedings of the Colloquium on Random Fields (Esztergom, Hungary, 1979), Colloq. Math. Soc. János Bolyai 27, North Holland, 1981, pp. 835-873.

[11] V. Pipiras and M. S. Taqqu, Integration questions related to fractional Brownian motion, Probab. Theory Related Fields 118 (2) (2000), pp. 251-291.

[12] M. S. Taqqu, Convergence of integrated processes of arbitrary Hermite rank, Z. Wahrsch. Verw. Gebiete 50 (1) (1979), pp. 53-83.

Atef Lechiheb

Université de Tunis El Manar

Faculté des sciences de Tunis

LR11ES13 Laboratoire d'Analyse stochastique

et applications

2092, Tunis, Tunisie

E-mail: atef.lechiheb@gmail.com

Guangqu Zheng

University of Kansas

Mathematics Department

Snow Hall, 1460 Jayhawk Blvd

Lawrence, Kansas 66045, USA

E-mail: gzheng90@ku.edu
Ivan Nourdin

Université du Luxembourg Unité de Recherche en Mathématiques

Maison du Nombre

6, avenue de la Fonte L-4364 Esch-sur-Alzette

Grand Duchy of Luxembourg E-mail: ivan.nourdin@uni.lu

Ezzedine Haouala

Université de Tunis El Manar Faculté des sciences de Tunis LR11ES13 Laboratoire d'Analyse stochastique et applications 2092, Tunis, Tunisie E-mail: ezdine.haouala@fst.rnu.tn

Received on 14.7.2016;

revised version on 18.2 .2017 